\title{
Signal and Noise Power Spectra in Superregenerative Oscillators
}

\author{
Jordi Bonet-Dalmau, Pere Palà-Schönwälder, F. Xavier Moncunill-Geniz, \\ Francisco del Águila-López, Rosa Giralt-Mas \\ Department of Mining, Industrial and ICT Engineering (EMIT), Manresa School of Engineering (EPSEM), \\ Universitat Politècnica de Catalunya (UPC), 08242 Manresa, Spain \\ jordi.bonet@upc.edu
}

\begin{abstract}
This paper presents a method to quantify noise in superregenerative oscillators. A frequency domain technique, originally intended to determine the signal response, can also be used to determine the noise response. This paper focuses on the procedure required to achieve this. Signal and noise spectra are obtained and their shape is compared. Finally, signal-to-noise ratio is computed for different quench signals.

Index Terms-Superregenerative oscillator, noise, frequency
\end{abstract} domain analysis, wireless receiver.

\section{INTRODUCTION}

$\mathbf{S}$ UPERREGENERATIVE (SR) receivers have lately caught renewed attention as a low-power and low-complexity receiver. Even if we have to go back as far as to 1922 [1] to see the birthday of the SR principle, the full understanding of the intricate relations between the input parameters and the performance of the SR receiver still poses a challenge, with new findings and applications being reported in the last years. For instance, the SR receiver has been extended to receive almost any type of modulation: analog and digital modulations, AM, FM [2], [3], and PM [4], [5] modulations, narrow-band and ultra-wide band modulations [6], with a variety of recent SR applications ranging from the IoT field [7] [8], to baseband amplification [9] or to an ultrasound wake-up receiver [10].

The analysis of SR receiver operation, in linear and logarithmic modes, can be made using different techniques. Analytical results can be obtained under some restrictions. Simulations have difficulties due to the different orders of magnitude between the RF signal and the quench signal, with envelopebased simulation techniques being most efficient for this kind of problem, in contrast with transient techniques. Harmonic balance methods can also be used to simulate the SR receiver when periodic excitations are used. Commercial simulators, such as ADS will rarely provide insight into the operation of the SR core. In contrast, the frequency domain technique presented in [11] can be used in order to understand the operation of the SR principles. This frequency domain method has been used to efficiently compute the relevant waveforms both in the time and frequency domains, including the exact envelope and the instantaneous phase and frequency of the generated responses to sinusoidal input signals.

Noise analysis is a relevant issue in communications receivers. Some remarks on the signal-to-noise ratio of SR

Work supported by Spanish Grant TEC2015-65748-R (MINECO/FEDER).

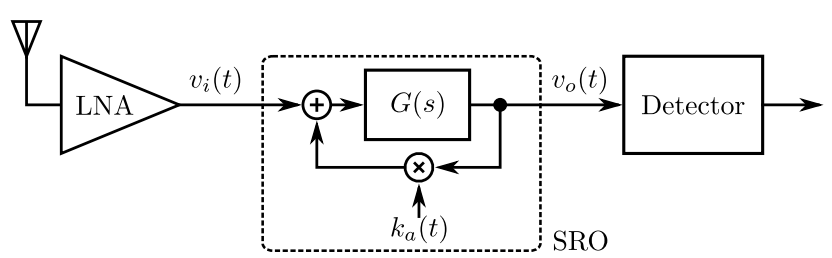

Fig. 1. Block diagram of the SR receiver.

circuits were already given in [2]. The response of a directsequence spread-spectrum SR receiver has been described in [12] basing on approximate analytic results. Closed form results for the SNR of a SR receiver were given in [13], also basing on approximate analytic results. More recently, the sensitivity of a SR oscillator (SRO) processing PSK signals has been investigated with a convolution model in the frequency domain [14], following the work in [15].

In this paper, the frequency domain technique [11] will be used to determine how the noise at the input, with a given spectral distribution, is transferred to the output of the SRO operating in the linear mode. The same method will be used to compute the output of the SRO given a sinusoidal input signal. This way, signal-to-noise ratio (SNR) will be easily computed.

\section{Frequency Domain Analysis}

The frequency domain technique [11] computes the output of a SRO for a sinusoidal input signal $v_{i}(t)$ of frequency $\omega_{i n}$, and a periodic (not necessarily sinusoidal [16]) quench signal $k_{a}(t)$ of frequency $\omega_{q}$ (see Fig. 1). The SR output $v_{o}(t)$ will be a combination of sinusoids, each one of frequency $\omega_{i n}+k \omega_{q}$, with $k$ integer. For the purpose of studying noise, this technique can also be applied when the input is a combination of sinusoids, each one of frequency $\omega_{i}=\omega_{i n}+i \omega_{q} / m$, with $i$ and $m$ integers. Each sinusoid of frequency $\omega_{i}$ will give an output as a combination of sinusoids, each one of frequency $\omega_{i, k}=\omega_{i}+k \omega_{q}$. The total output will be computed applying superposition, under the restriction of linear mode of operation, i.e. adding the particular outputs of each sinusoid at the input.

\section{Noise Computation Procedure}

Our goal is to compute the power spectral density (PSD) of noise at the output of the SRO when the PSD of noise 
at the input is known. In order to present the main ideas we will consider white noise at the input, i.e. a constant doublesided PSD of value $\eta / 2$. This value is computed assuming that the SR receiver has a LNA (of $0 \mathrm{~dB}$ gain) as a front end that matches the impedance $R$ of the source and with an ideal voltage controlled source at the output, connected to the SRO input. In the following we will consider $\eta / 2=R k_{B} T / 2$, with $R=50 \Omega, k_{B}$ the Boltzmann constant and $T=290 \mathrm{~K}$. This value is approximately

$$
\eta / 2=1 \times 10^{-19} \mathrm{~V}^{2} / \mathrm{Hz} \text { or }-190 \mathrm{dBV} / \mathrm{Hz} .
$$

In order to use the previously described frequency domain technique, we will transfer the white noise power (with $\mathrm{PSD}=$ $\eta / 2)$ present in each bandwidth $\Delta \omega$ (in rad), i.e. $\frac{\eta}{2} \frac{\Delta \omega}{2 \pi}$, onto a train of impulses spaced at intervals of $\Delta \omega$. So, to preserve the total power, the amplitude $A$ of each impulse is chosen in a way that its power is equal to

$$
A^{2}=\frac{\eta}{2} \frac{\Delta \omega}{2 \pi} .
$$

This procedure is exact when $\Delta \omega \rightarrow 0$.

Figure 2 and 3 show the output power spectra due to impulses at some selected frequencies. Each impulse generates an output spectrum with a similar envelope shape. When the impulse frequency $\omega_{i}$ is equal to $\omega_{0}$, the center frequency of the selective network

$$
G(s)=k_{0} \frac{s \omega_{0} / Q_{0}}{s^{2}+s \omega_{0} / Q_{0}+\omega_{0}^{2}}
$$

of the SRO in Fig. 1, the amplitude of this envelope is maximum. As expected, each impulse at frequency $\omega_{i}$ generates an output with impulses at frequencies $\omega_{i}+k \omega_{q}$. So, impulses separated by a multiple of $\omega_{q}$ will give spectra that overlap. This overlap has been avoided in Fig. 2 and 3, thanks to the chosen values of the impulse frequencies, to clearly show the output spectrum due to each input.

As we have chosen $\Delta \omega=\omega_{q} / m$ to ease the procedure, some spectra will overlap. The way to deal with this overlap is straightforward thanks to the statistical properties of noise. Instead of repeatedly computing the output signal spectrum generated by a train of impulses with random phase, to compute the total output power spectrum from the mean of each output signal spectrum, we can simply add the output power spectrum generated by each of the impulses of a train of impulses, which can have an arbitrary phase.

As a result of this procedure we have an output power spectrum made of impulses at frequency $\omega_{i^{\prime}}$ with power $\left|C_{i^{\prime}}\right|^{2}$. Now we reverse the initial transfer of power by distributing the power of each impulse over the bandwidth $\Delta \omega$ around its frequency. This way, the final double-sided $\operatorname{PSD}(\omega)$ in the interval $\omega_{i^{\prime}}-\Delta \omega / 2 \leq \omega<\omega_{i^{\prime}}+\Delta \omega / 2$ is constant an equal to

$$
\operatorname{PSD}\left(\omega_{i^{\prime}}\right)=\left|C_{i^{\prime}}\right|^{2} \frac{2 \pi}{\Delta \omega},
$$

where $\left|C_{i^{\prime}}\right|^{2}$ is measured in $\mathrm{V}^{2}$ and PSD in $\mathrm{V}^{2} / \mathrm{Hz}$.

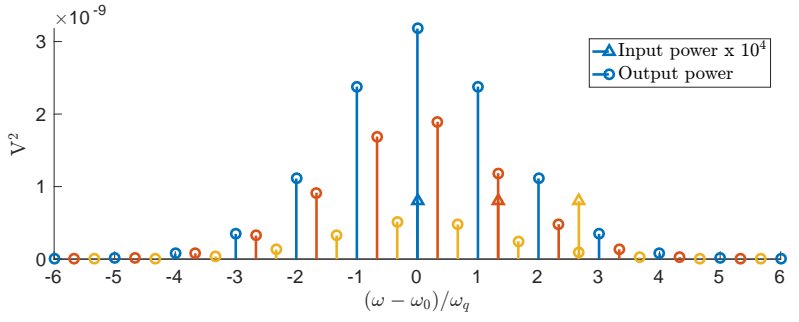

Fig. 2. SR output power spectrum in $\mathrm{V}^{2}$ due to impulses at frequencies $\omega_{0}$ (blue), $\omega_{0}+4 \omega_{q} / 3$ (red) and $\omega_{0}+8 \omega_{q} / 3$ (yellow).

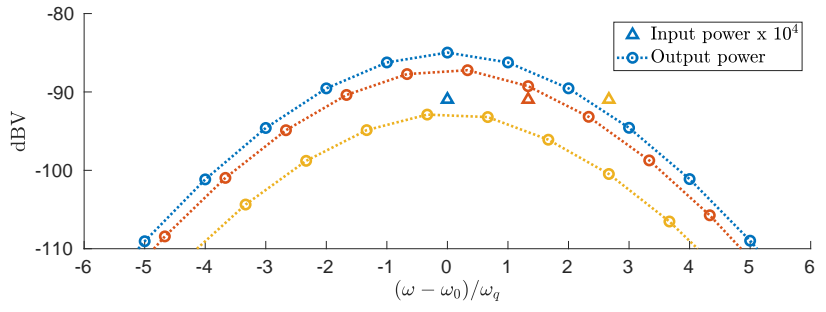

Fig. 3. SR output power spectrum in $\mathrm{dBV}$ due to impulses at frequencies $\omega_{0}$ (blue), $\omega_{0}+4 \omega_{q} / 3$ (red) and $\omega_{0}+8 \omega_{q} / 3$ (yellow).

\section{NoIse SPECTRUM}

Next we will follow the procedure explained in the previous section to compute the noise PSD at the output of the SRO when at its input we have white noise with $\mathrm{PSD}=\eta / 2$. First we consider a frequency impulse train at intervals $\Delta \omega=$ $\omega_{q} / m$

$$
A(\omega)=\sum_{i=-K_{1}}^{i=K_{2}} A \delta\left(\omega-\omega_{i}\right),
$$

with $A$ given by (2) and

$$
\omega_{i}=\omega_{0}+i \Delta \omega=\omega_{0}+\frac{i}{m} \omega_{q} .
$$

As the input is band-pass filtered by $G(s)$, with bandwidth $\mathrm{BW}=\omega_{0} / Q_{0}$, we have limited the input noise in the frequency range around $\omega_{0}$, i.e $\omega_{0}+K_{2} \Delta \omega \approx \omega_{0}+r \mathrm{BW}=\omega_{0}\left(1+r / Q_{0}\right)$ and $\omega_{0}-K_{1} \Delta \omega \approx \omega_{0} /\left(1+r / Q_{0}\right)$. We have found no relevant differences in the results when $r>3$. Notice that only the positive part of the spectrum is considered in (5).

Next, we consider a periodic quench signal $k_{a}(t)$ of frequency $\omega_{q}$ whose spectrum is

$$
B(\omega)=\sum_{l=-L}^{l=L} B_{l} \delta\left(\omega-l \omega_{q}\right),
$$

with $B_{-l}=B_{l}^{*}$. Now we can solve the linear system of equations given by [11]

$$
\mathbf{c}-\mathbf{G a}-\mathbf{G D c}=\mathbf{0}
$$

for each one of the impulses in (5), of amplitude $A$ and frequency $\omega_{i}$, that will give the output

$$
C_{i}(\omega)=\sum_{n=-N_{1}}^{n=N_{2}} C_{i, n} \delta\left(\omega-\omega_{i, n}\right) .
$$


where

$$
\omega_{i, n}=\omega_{i}+n \omega_{q},
$$

and $N_{1}$ and $N_{2}$ limit the frequency range of the output spectrum. Substituting (6) in (10) we have

$$
\omega_{i, n}=\omega_{0}+\left(\frac{i}{m}+n\right) \omega_{q}=\omega_{0}+\frac{i^{\prime}}{m} \omega_{q}=\omega_{i^{\prime}},
$$

with

$$
i^{\prime}=i+m n \text {. }
$$

Notice that various combinations of $i$ and $n$ gives the same $i^{\prime}$, and the range of $i^{\prime}$ is

$$
-K_{1}-m N_{1} \leq i^{\prime} \leq K_{2}+m N_{2} .
$$

The total output power $\left|C_{i^{\prime}}\right|^{2}$ at each frequency $\omega_{i^{\prime}}$ will be computed taking into account (9) for each $i$, i.e. adding the power of all the coefficients $C_{i, n}$ whose frequencies $\omega_{i, n}$ coincide:

$$
\left|C_{i^{\prime}}\right|^{2}=\sum_{i^{\prime}=i+m n}\left|C_{i, n}\right|^{2}
$$

As our goal is to compute the output spectrum in the same range as the input $A(\omega)$ in (5), we have chosen the summation limits in (9) to be $N_{1}=N_{2}=N \geq\left(\omega_{K_{2}}-\omega_{-K_{1}}\right) / \omega_{q}=$ $\left(K_{2}+K_{1}\right) / m$. In addition, we only consider the output spectrum in the range

$$
\omega_{-K_{1}} \leq \omega_{i^{\prime}} \leq \omega_{K_{2}}
$$

to avoid discontinuities caused by the finite value of $N$ in the output spectrum. Equation (15) means that instead of the range in (13), $i^{\prime}$ will take the truncated range

$$
-K_{1} \leq i^{\prime} \leq K_{2}
$$

So, the output power at each frequency can be extracted from

$$
C_{\text {noise }}(\omega)=\sum_{i^{\prime}=-K_{1}}^{i^{\prime}=K_{2}} C_{i^{\prime}} \delta\left(\omega-\omega_{i^{\prime}}\right) .
$$

Finally, the total SRO noise output power taking into account all the spectrum, positive and negative frequencies, is given by

$$
P_{\text {noise }}=2 \sum_{i^{\prime}=-K_{1}}^{i^{\prime}=K_{2}}\left|C_{i^{\prime}}\right|^{2} .
$$

Figure 4 shows the envelope of the power of the coefficients $C_{i^{\prime}}$ at frequencies $\omega_{i^{\prime}}$ compared with the open-loop output, in which the output is directly the input filtered out by $G(s)$. Using the transformation (4) we compute the PSD of noise shown in Fig. 5. Both figures have been obtained using the following set of parameters: a selective network (3) with $k_{0}=$ $1, Q_{0}=50$ and $f_{0}=2.4 \mathrm{GHz}$, a sinusoidal quench signal (7) with $f_{q}=2.4 \mathrm{MHz}, B_{0}=0.8$ and $B_{1}=0.2465$, and white noise at the input with a PSD given by (1). With this set of parameters the SRO has an amplification factor $K=60 \mathrm{~dB}$. This amplification factor $K$ is the peak amplitude gain of the envelope of the SR output pulse $v_{o}(t)$ referred to the amplitude

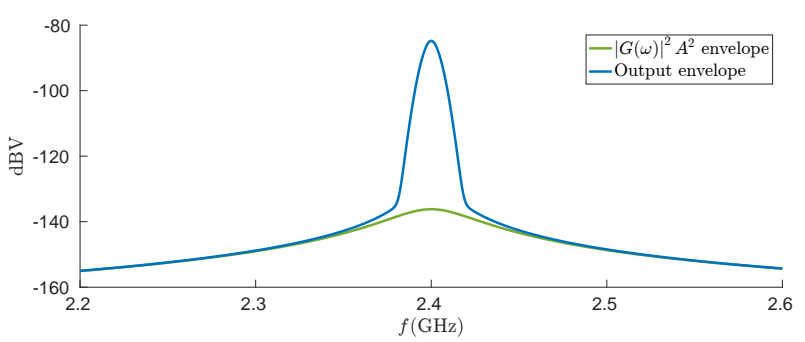

Fig. 4. Envelope of the noise power spectrum in $\mathrm{dBV}$ at the output of the SRO due to the impulse train (5) for a sinusoidal quench.

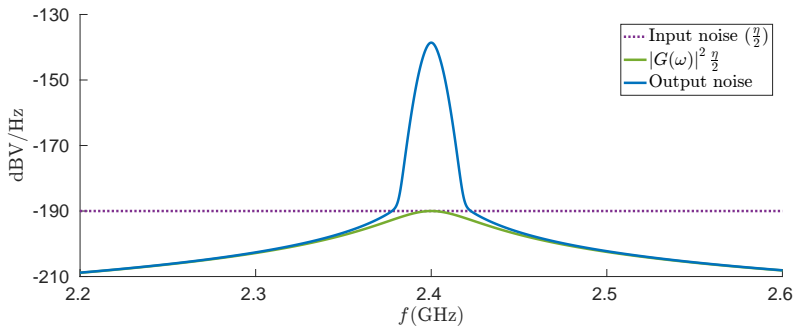

Fig. 5. PSD of noise in $\mathrm{dBV} / \mathrm{Hz}$ at the output of the $\mathrm{SRO}$ due to white noise of $\mathrm{PSD}=\frac{\eta}{2}$ for a sinusoidal quench.

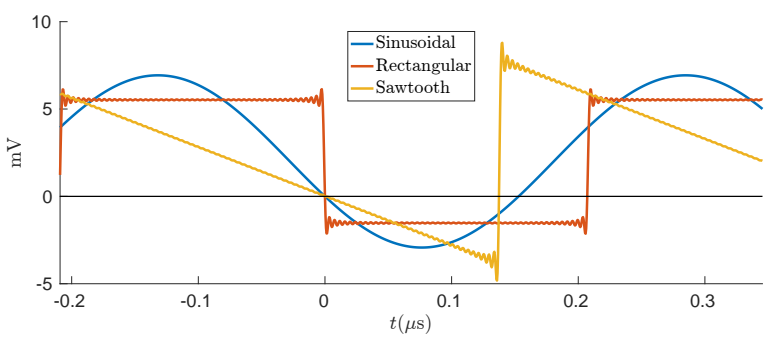

Fig. 6. Instantaneous damping factor $\xi(t)$ for sinusoidal, rectangular and sawtooth quench signals $k_{a}(t)$. The last two take $L=101$ harmonics in (7).

of a sinusoidal signal at the input $v_{i}(t)$, which is different from the power gain. According to the value of $m$, that has been chosen equal to 10 , i.e $\Delta \omega=\omega_{q} / 10$, the difference between Fig. 4 and Fig. 5 is $10 * \log _{10}\left(\frac{2 \pi}{\Delta \omega}\right)=-53.80 \mathrm{~dB}$. In order to observe the dependence of the noise spectrum with the shape of the quench signal, we have repeated the previous computations with the same set of parameters, but changing the sinusoidal quench by a rectangular and a sawtooth quench. The parameters of each quench signal have been tuned in order to maintain the hangover effect: $B_{0}$ in (7) is constant, and the amplification factor is fixed to $K=60 \mathrm{~dB}$ (to achieve this result the peak-to-peak amplitude of the rectangular quench is 0.7049 and that of the sawtooth quench is 1.1693). Figure 6 shows the instantaneous damping factor

$$
\xi(t)=\frac{1-k_{0} k_{a}(t)}{2 Q_{0}}
$$

for each one of the quench signals $k_{a}(t)$. We observe the Gibbs phenomenon in the rectangular and sawtooth quenches due to the truncation of the Fourier series: $L=101$ harmonics in (7). Figure 7 shows the results for these three types of quench signal. The total SRO noise output power computed using (18) is: $-66.4 \mathrm{dBV}$ for a sinusoidal quench, $-69.2 \mathrm{dBV}$ for a rectangular quench and $-74.4 \mathrm{dBV}$ for a sawtooth quench. 


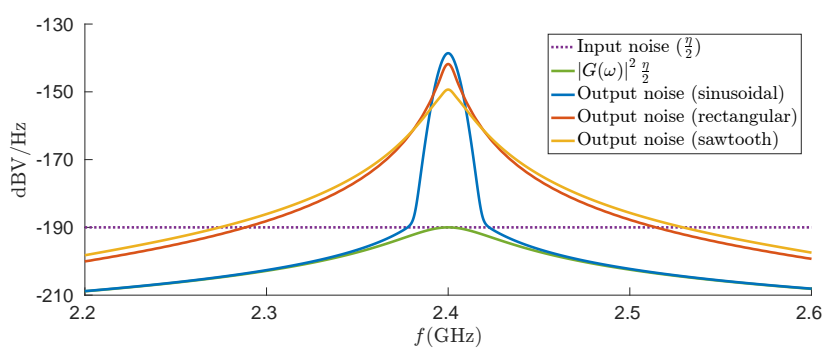

Fig. 7. PSD of noise in $\mathrm{dBV} / \mathrm{Hz}$ at the output of the $\mathrm{SRO}$ due to white noise of $\mathrm{PSD}=\frac{\eta}{2}$ for each of the quench signals $k_{a}(t)$ in Fig. 6.

\section{Signal Spectrum}

In this section we will compute the spectrum due to a continuous sinusoidal signal tuned to the selective network $G(s)$, i.e. of frequency $\omega_{0}$. Results of the previous chapter obtained to compute noise are easily particularized for $i=0$. For a single impulse of frequency $\omega_{0}$ and amplitude $A_{0}(5)$, becomes

$$
A(\omega)=A_{0} \delta\left(\omega-\omega_{0}\right)
$$

and (9) becomes

$$
C_{0}(\omega)=\sum_{n=-N_{1}}^{n=N_{2}} C_{0, n} \delta\left(\omega-\omega_{0, n}\right) .
$$

or, in a more friendly notation,

$$
C_{0}(\omega)=\sum_{n=-N_{1}}^{n=N_{2}} C_{n} \delta\left(\omega-\left(\omega_{0}+n \omega_{q}\right)\right) .
$$

In order to study the signal spectrum in the same range as the noise spectrum, we will consider $\omega_{0}+n \omega_{k}$ in the range given by (15), i.e.

$$
-K_{1} / m \leq n \leq K_{2} / m
$$

This way, the signal spectrum is given by

$$
C_{\text {signal }}(\omega)=\sum_{n=-n_{1}}^{n=n 2} C_{n} \delta\left(\omega-\left(\omega_{0}+n \omega_{q}\right)\right),
$$

where $-n_{1}$ and $n_{2}$ are the lower and upper limits of $n$ in (23). The total SRO signal output power taking into account all the spectrum is given by

$$
P_{\text {signal }}=2 \sum_{n=-n_{1}}^{n=n_{2}}\left|C_{n}\right|^{2} .
$$

Notice that the components $C_{n}$ of the signal spectrum (24) are separated $\omega_{q}$ while the components $C_{i^{\prime}}$ of the noise spectrum (17) are separated $\Delta \omega=\omega_{q} / m$. Fig. 8 shows the power of each coefficient $C_{n}$ in (24) for each one of the quench signals previously used. The power of the input signal has chosen to be $-80 \mathrm{dBm}$ over a resistance of $50 \Omega$, i.e. $-93.0 \mathrm{dBV}$. So, $A_{0}^{2}=2.5 \times 10^{-10} \mathrm{~V}^{2}$. The total SRO signal output power computed using (25) is $-41.6 \mathrm{dBV}$ (a power gain of $51.4 \mathrm{~dB}$ ) for a sinusoidal quench, $-44.8 \mathrm{dBV}$ (a power gain of $48.2 \mathrm{~dB}$ ) for a rectangular quench and $-48.1 \mathrm{dBV}$ (a

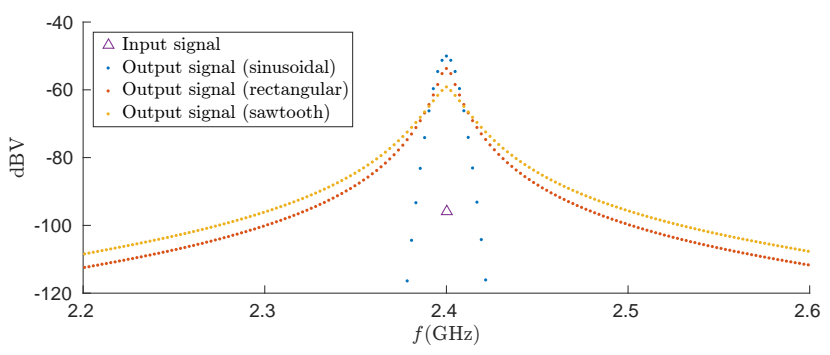

Fig. 8. Signal power spectrum in $\mathrm{dBV}$ at the output of the SRO due to a sinusoidal signal for each of the quench signals $k_{a}(t)$ in Fig. 6.

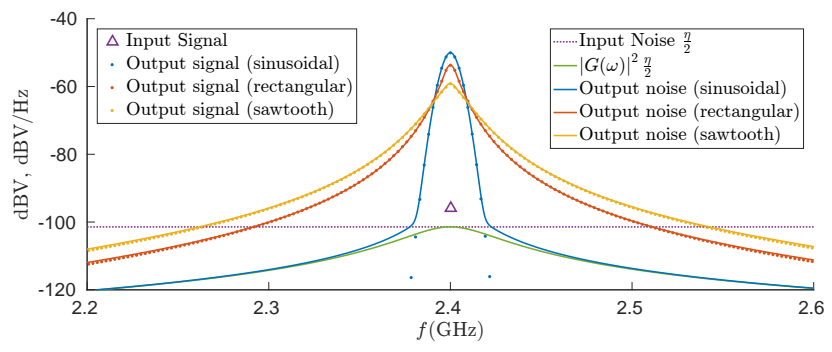

Fig. 9. PSD of noise in $\mathrm{dBV} / \mathrm{Hz}$ and signal power spectrum in $\mathrm{dBV}$ at the output of the SRO for each of the quench signals $k_{a}(t)$ in Fig. 6. For each quench signal, the PSD of noise has been scaled to make its maximum value in $\mathrm{dBV} / \mathrm{Hz}$ equal to the maximum value of the signal power spectrum in $\mathrm{dBV}$.

power gain of $44.9 \mathrm{~dB}$ ) for a sawtooth quench. This power gain is lower that the peak amplitude gain $K=60 \mathrm{~dB}$. Fig. 9 plots noise and signal spectra simultaneously. The shape of the envelope of the signal spectrum is almost equal to the noise PSD for the rectangular and sawtooth quenches. This is also true for the sinusoidal quench at frequencies close to $\omega_{0}$, while, when moving away from $\omega_{0}$, the signal spectrum doesn't flatten to follow the frequency response of the selective network $G(s)$, showing a much narrower spectrum than that of noise. The SNR at the output of the SRO is computed in a straightforward form from (18) and (25):

$$
S N R=\frac{P_{\text {signal }}}{P_{\text {noise }}}=\frac{\sum_{n=-n_{1}}^{n=n_{2}}\left|C_{n}\right|^{2}}{\sum_{i^{\prime}=-K_{1}}^{i^{\prime}=K_{2}}\left|C_{i^{\prime}}\right|^{2}} .
$$

The SNR is $24.8 \mathrm{~dB}$ for a sinusoidal quench, $24.3 \mathrm{~dB}$ for a rectangular quench and $26.3 \mathrm{~dB}$ for a sawtooth quench. The difference between the best SNR, given by the sawtooth quench, and the worst SNR, given by the rectangular quench, is just $2 \mathrm{~dB}$.

\section{CONCLUSION}

A procedure to study the PSD of noise in a SRO operating in the linear mode has been presented. The method is based on transferring the input noise power present in a small bandwidth onto an impulse. Then, the output spectrum for each single impulse is computed using a frequency domain technique. Finally, the overall response is computed applying superposition, considering some statistical properties of noise, and distributing the power of each output impulse over a small bandwidth around its frequency. SNR results for different quench signals have been obtained. 


\section{REFERENCES}

[1] E. Armstrong, "Some recent developments of regenerative circuits," Proceedings of the Institute of Radio Engineers, vol. 10, no. 4, pp. 244260, Aug 1922.

[2] H. P. Kalmus, "Some notes on superregeneration with particular emphasis on its possibilities for frequency modulation," Proceedings of the IRE, vol. 32, no. 10, pp. 591-600, 1944.

[3] P. Pala-Schonwalder, J. Bonet-Dalmau, A. Lopez-Riera, F. MoncunillGeniz, F. del Aguila-Lopez, and R. Giralt-Mas, "Superregenerative reception of narrowband FSK modulations," Circuits and Systems I: Regular Papers, IEEE Transactions on, vol. 62, no. 3, pp. 791-798, Mar 2015.

[4] L. Hernandez and S. Paton, "A superregenerative receiver for phase and frequency modulated carriers," in Circuits and Systems, 2002. ISCAS 2002. IEEE International Symposium on, vol. 3, 2002, pp. III-81-III84 vol.3.

[5] P. Pala-Schonwalder, J. Bonet-Dalmau, F. Xavier Moncunill-Geniz, F. del Aguila-Lopez, and R. Giralt-Mas, "A superregenerative QPSK receiver," Circuits and Systems I: Regular Papers, IEEE Transactions on, vol. 61, no. 1, pp. 258-265, Jan 2014.

[6] M. Pelissier, D. Morche, and P. Vincent, "Super-regenerative architecture for UWB pulse detection: From theory to RF front-end design," IEEE Transactions on Circuits and Systems I: Regular Papers, vol. 56, no. 7, pp. 1500-1512, July 2009.

[7] A. López-Riera, F. del Águila López, P. Palá-Schönwälder, J. BonetDalmau, R. Giralt-Mas, and F. X. Moncunill-Geniz, "Joint symbol and chip synchronization for a burst-mode-communication superregenerative MSK receiver," IEEE Transactions on Circuits and Systems I: Regular Papers, vol. 64, no. 5, pp. 1260-1269, May 2017.

[8] M. Anis, M. Ortmanns, and N. Wehn, "A $2.5 \mathrm{mw} 2 \mathrm{mb} / \mathrm{s}$ fully integrated impulse-FM-UWB transceiver in $0.18 \mu \mathrm{m}$ CMOS," in 2011 IEEE MTT-S International Microwave Symposium, June 2011, pp. 1-3.

[9] R. Rieger and N. Sulistiyanto, "Integrated circuit for super-regenerative low-frequency amplification," IEEE Transactions on Circuits and Systems II: Express Briefs, vol. 65, no. 1, pp. 31-35, Jan 2018.

[10] H. Fuketa, S. O'uchi, and T. Matsukawa, "A $0.3-\mathrm{V} 1-\mu \mathrm{W}$ superregenerative ultrasound wake-up receiver with power scalability," IEEE Transactions on Circuits and Systems II: Express Briefs, vol. 64, no. 9, pp. 1027-1031, Sept 2017.

[11] J. Bonet-Dalmau, F. Moncunill-Geniz, P. Pala-Schonwalder, F. del Aguila-Lopez, and R. Giralt-Mas, "Frequency domain analysis of superregenerative receivers in the linear and the logarithmic modes," Circuits and Systems I: Regular Papers, IEEE Transactions on, vol. 59, no. 5, pp. 1074-1084, May 2012.

[12] F. Moncunill-Geniz and P. Pala-Schonwalder, "Performance of a DSSS superregenerative receiver in the presence of noise and interference," in Circuits and Systems, 2006. ISCAS 2006. Proceedings. 2006 IEEE International Symposium on, May 2006, pp. 5687-5690.

[13] P. Thoppay, C. Dehollaini, and M. Declercq, "Noise analysis in superregenerative receiver systems," in Research in Microelectronics and Electronics, 2008. PRIME 2008. Ph.D., Jun 2008, pp. 189-192.

[14] D. G. Lee and P. P. Mercier, "Noise analysis of phase-demodulating receivers employing super-regenerative amplification," IEEE Transactions on Microwave Theory and Techniques, vol. 65, no. 9, pp. 3299-3311, Sept 2017.

[15] J. Bohorquez, A. Chandrakasan, and J. Dawson, "Frequency-domain analysis of super-regenerative amplifiers," Microwave Theory and Techniques, IEEE Transactions on, vol. 57, no. 12, pp. 2882-2894, Dec 2009.

[16] P. Palà-Schönwälder, J. Bonet-Dalmau, F. del Águila López, R. Sanahuja, and F. X. Moncunill-Geniz, "Effects of quench discretization on superregenerative oscillators," in 2012 IEEE International Symposium on Circuits and Systems, May 2012, pp. 1303-1306. 